\title{
THE LIFE AND WORK OF KUO-TSAI CHEN
}

Kuo-Tsai Chen was born on July 15, 1923 in Chekiang, China. He earned a Bachelor of Science degree in mathematics from Southwest Associated University in Kungming in 1946. He then moved to Shanghai, where he became an Assistant at the Mathematics Institute of the Academia Sinica in 1946-47. On the recommendation of its director, Shing-Shen Chern, he went to work with Samuel Eilenberg at Indiana University. After one year there, he followed Eilenberg to Columbia University in New York, where he received his doctorate in 1950. During his graduate studies, he was a mathematics instructor at the National Bible Institute in New York from 1948 to 1950, and an Assistant at Columbia University in 1949-50. After being awarded his Ph.D. degree, he went first to Princeton University as an instructor in 1950-51, and then to the University of Illinois as a Research Associate in 1951-52.

His next position was that of a Lecturer at the University of Hong Kong where he stayed from 1952 to 1958 . His parents were then living in Taipeh, Taiwan. In the first course he gave in Hong Kong, Chester Chen, as he had become known, met a charming sophomore, Julia Tse-Yee Fong, who became his bride in 1953. His very strict sense of duty did not allow him to give his preferred student special help, which occasionally made her very mad at him. Their happy marriage brought forth three children: Matthew in 1955, who earned a Doctorate in mathematics at the University of California at Berkeley and who is currently an electrical engineer with AT \& T; Lydia in 1956, who graduated from Sarah Lawrence College, and is now a painter and editor; Lucia in 1960, who graduated from MIT and is now a graduate student in material science at the University of Illinois.

Chen's next position was at the Instituto Tecnologico de Aeronautica in Sao Jose dos Campos, Brazil, first as an Associate Professor in 1958-59, and then as a Professor in 1959-60. He became a member of the Institute for Advanced Study in Princeton in the winter of 1960-61, and again later in the spring of

\footnotetext{
${ }^{1}$ It is in fact the inverse limit of the nilpotent Lie group $\&(A / I) / \mathbb{S}(A / I) \cap\left(1+J^{n}\right)$. These are simply connected as they are diffeomorphic to their Lie algebras $g(A / I) / \mathfrak{g}(A / I) \cap J^{n}$ via the exponential map.
} 
1962, the fall of 1971 , and finally in the spring of 1979 . He was appointed Associate Professor at Rutgers University in 1962-63, and became a Full Professor there from 1963 to 1965 . From there he went to the State University of New York in Buffalo where he stayed from 1965 to 1967. In 1967 he once again joined the faculty of the University of Illinois at Urbana, this time as a Professor. He remained there until his death in August, 1987 which followed a long illness. He was a devout Christian throughout his life.

Chen was an outstanding and original mathematician. His work falls naturally into three periods: his early work on group theory and links in the three sphere; his subsequent work on formal differential equations, which gradually developed into his most powerful and important work; his work on iterated integrals and homotopy theory, which occupied him for the last twenty years of his life. The goal of Chen's iterated integrals program, which is a de Rham theory for path spaces, was to study the interaction of topology and analysis through path integration.

Chen's early work contains significant contributions to the theory of links in the three sphere. Two smooth links are said to be isotopic if the associated imbeddings of $S^{1}$ into $S^{3}$ can be deformed, one into the other, through smooth imbeddings. In [3] Chen showed that the quotients

$$
\pi^{1} / \pi^{s+1}
$$

of the lower central series

$$
\pi_{1}\left(S^{3}-L\right)=\pi^{1} \supset \pi^{2} \supset \pi^{3} \supset
$$

of the link group depend only on the isotopy class of the link. Since all knots are isotopic to the trivial knot, one obtains as a special case the well known result that the lower central series of a knot group stabilizes at $\pi^{2}$ :

$$
\pi^{s}=\pi^{2} \text { for all } s>2 .
$$

This led to Milnor's work on isotopy invariants of links, notably his definition of $\bar{\mu}$-invariants, which are numerical invariants of the lower central series of a link group and are generalized linking numbers $[\mathrm{M}]$.

Even though Chen, in collaboration with Fox and Lyndon, did give an algorithm for computing the quotients of the lower central series of a finitely presented group [10], the problem of computing its quotients remains extremely difficult. In his thesis [1], Chen showed that for any finitely presented group $\pi$, the quotients of the lower central series of $\pi / \pi^{\prime \prime}$ are computable, where $\pi^{\prime \prime}$ denotes the second derived subgroup $\left[\pi^{2}, \pi^{2}\right]$ of $\pi$. When $\pi$ is a link group, these groups are now known as the Chen groups of the link and are isotopy invariants.

Chen's subsequent work is clearly united by the dual threads of formal Lie theory and the theory of connections on bundles whose structure group is a 
"Lie group" of formal power series. The germ of these ideas first appears in his very first paper on group theory [1], although it becomes clearer through the papers [5], [7]-[9], [11]-[21], while from [22] on, iterated integrals become the main theme of his work. To illustrate Chen's original ideas, we present a somewhat revisionist view of his approach to de Rham homotopy theory through formal Lie theory which he developed at about the same time as Dennis Sullivan developed his theory of minimal models [S1], [S2].

Formal Lie theory takes place in a formal power series ring: Denote the ring of formal power series in the non-commuting indeterminates $X_{1}, \ldots, X_{n}$ over $\mathbf{R}$ by

$$
A=\mathbf{R}\left\langle\left\langle X_{1}, \ldots, X_{n}\right\rangle\right\rangle
$$

This is a complete topological ring. The neighborhoods of 0 are the powers of the maximal ideal

$$
J=\{\text { power series with trivial constant term }\} .
$$

It can be viewed as a Lie algebra with bracket

$$
[U, V]=U V-V U \text {. }
$$

Let $\mathbf{L}\left(X_{1}, \ldots, X_{n}\right)$ denote the Lie sub-algebra of $A$ generated by $X_{1}, \ldots, X_{n}$ and its closure in $A$ by

$$
\mathfrak{g}(A)=\mathbf{L}\left(X_{1}, \ldots, X_{n}\right)^{\wedge} .
$$

The exponential and logarithm maps

$$
\exp : J \rightarrow 1+J, \quad \log : 1+J \rightarrow J,
$$

defined using the usual power series, are continuous and mutually inverse. The prototypical example of a "Lie group" of formal power series, called a Malcev group in the literature (cf. [Q]), is

$$
\mathbb{E S}(A)=\{X \in A: \log X \in \mathfrak{g}(A)\} .
$$

This is an infinite dimensional Lie group with Lie algebra $g(A)$. All other Malcev groups are obtained by replacing $A$ by $A / I$, where $I$ is a closed ideal of $A$ generated by elements of $\mathfrak{g}(A)$. One then defines $\mathfrak{g}(A / I)$ to be the image of $\mathfrak{g}(A)$ in $A / I$ and $\mathbb{S}(A / I)$ to be the image of $\mathfrak{S}(A)$. Again, the exponential map $\mathfrak{g}(A / I) \rightarrow \mathbb{S}(A / I)$ is an isomorphism, so that $\mathbb{B}(A / I)$ behaves like a simply connected nilpotent Lie group. ${ }^{1}$

\footnotetext{
${ }^{1}$ It is in fact the inverse limit of the nilpotent Lie group $(S)(A / I) / \circlearrowleft(A / I) \cap\left(1+J^{n}\right)$. These are simply connected as they are diffeomorphic to their Lie algebras $\mathfrak{g}(A / I) / \mathfrak{g}(A / I) \cap J^{n}$ via the exponential map.
} 
Chen's basic tool is the transport map of a trivialized bundle. Let $M$ be a manifold, assumed to be smooth as throughout this article. A trivialized principal $G$-bundle over $M$ is a trivial bundle $M \times G \rightarrow M$ together with a distinguished trivialization, unique up to the right action of $G$. Here $G$ may be a subgroup of $G L(n)$ or a Malcev group. A connection on a trivialized bundle corresponds to a $\mathfrak{g}$-valued 1 -form $\omega$ on $M$ via the rule

$$
\nabla s=d s-s \omega
$$

where $s: M \rightarrow G$ is a section. Denote the space of piecewise smooth paths $[0,1] \rightarrow M$ by $P M$. Given a connection $\omega$ on a tivialized bundle, we obtain a transport map

$$
T_{\omega}: P M \rightarrow G
$$

which takes the path $\gamma$ to the result of parallel transporting the identity along $\gamma$. Equivalently, $T(\gamma)=X(1)$, where $X(t)$ is the solution of the initial value problem

$$
X^{\prime}(t)=X(t) A(t), \quad X(0)=I d
$$

Here $A:[0,1] \rightarrow \mathfrak{g}$ is the function defined by

$$
\gamma^{*} \omega=A(t) d t
$$

From elementary differential geometry, we know that whenever $\alpha$ and $\beta$ are composable paths, $T(\alpha) T(\beta)=T(\alpha \beta)$.

When $G$ is a Malcev group, equation (2) is what Chen called a formal differential equation; cf. [12]-[20]. These were a key ingredient in his earlier work on normal forms of germs of diffeomorphisms $\left(\mathbf{R}^{n}, 0\right) \rightarrow\left(\mathbf{R}^{n}, 0\right)$ and their infinitesimal analogue, germs of vector fields at 0 in $\mathbf{R}^{n}$. In [15], [17], [18], [19], [21], [24] Chen studied the behaviour of the integral curves of a vector field near a singular point, and the behaviour of a local diffeomorphism near a fixed point building on a line of thought that had originated with Sternberg. One of the main results is a nonlinear decomposition theorem for germs of diffeomorphisms analogous to the semi-simple times unipotent decomposition of matrices [17]. For vector fields he established the infinitesimal analogue in [18], where he showed that the germ of a vector field with an elementary critical point at 0 has a Jordan canonical form; that is, it can be written as the sum of commuting semi-simple and nilpotent vector fields. He used this to show that two vector fields, each with an elementary critical point at the origin, are equivalent if and only if they are formally equivalent. The corresponding result for diffeomorphisms is proved in [19].

The cornerstone of Chen's work in homotopy theory is a "well known" but elegant formula for $T_{\omega}$. To express it, we need to introduce Chen's iterated 
integrals which he introduced in [5] in a special case, in [7] for 1-forms, and in [32] for higher dimensional forms. Suppose that $w_{1}, \ldots, w_{r}$ are smooth forms on $M$ taking values in an associative algebra $A$ (e.g., $\mathfrak{g} \mathfrak{I}(n), U \mathfrak{g}$ or a power series ring), each of degree at least 1 , then

$$
\int w_{1} w_{2} \ldots w_{r}
$$

is an $A$-valued differential form on $P M$ of degree

$$
\sum_{1}^{r}\left(\operatorname{deg} w_{j}-1\right)
$$

Representing the standard $r$-simplex as

$$
\Delta^{r}=\left\{\left(t_{1}, \ldots, t_{r}\right) \in \mathbf{R}^{n}: 0 \leq t_{1} \leq \cdots \leq t_{r} \leq 1\right\},
$$

define a smooth function $\Phi: \Delta^{r} \times P M \rightarrow M^{r}$ by

$$
\Phi\left(\left(t_{1}, \ldots, t_{r}\right), \gamma\right)=\left(\gamma\left(t_{1}\right), \ldots, \gamma\left(t_{r}\right)\right) .
$$

The iterated integral is then defined by

$$
\int w_{1} w_{2} \cdots w_{r}=\pi_{*} \Phi^{*}\left(w_{1} \times w_{2} \times \cdots \times w_{r}\right),
$$

where $\pi$ denotes the projection of $\Delta^{r} \times P M$ onto $P M$ and $\pi_{*}$ denotes integration over the fiber of $\pi$ with respect to the volume form $d t_{1} \wedge \cdots \wedge d t_{r}$.

When each $w_{j}$ is a 1 -form, $\int w_{1} w_{2} \ldots w_{r}$ is a function

$$
\int w_{1} w_{2} \ldots w_{r}: P M \rightarrow A
$$

which is a very natural generalization of the usual line integral. It takes the path $\gamma$ to

$$
\underset{0 \leq t_{1} \leq \ldots \leq t_{r} \leq 1}{ } f_{1}\left(t_{1}\right) \ldots f_{r}\left(t_{r}\right) d t_{1} \ldots d t_{r}
$$

where $g^{*} w_{j}=f_{j}(t) d t$.

The transport formula is obtained by solving (2) by Picard iteration. It is analogous to the Dyson exponential of physics.

Lemma (Chen [41], [12], [7]). Suppose that $G$ is a Lie or Malcev group with Lie algebra $\mathrm{g}$. If the g-valued 1-form $\omega$ defines a connection on the bundle 
$M \times G \rightarrow M$ via(1), then the transport is given by the convergent power series

$$
T_{\omega}(\gamma)=1+\int_{\gamma} \omega+\int_{\gamma} \omega \omega+\int_{\gamma} \omega \omega \omega+\ldots
$$

A connection $\omega$ on a trivialized bundle is flat if and only if its curvature vanishes:

$$
d \omega+1 / 2[\omega, \omega]=0 .
$$

In this case the value of the transport on a path depends only on its homotopy class. It induces a holonomy (or monodromy) homomorphism

$$
\Theta: \pi_{1}(M, x) \rightarrow G, \quad\{\gamma\} \rightarrow T(\gamma) .
$$

To see what one can do with this, consider a manifold $M$ which possesses closed 1-forms $w_{1}, \ldots, w_{r}$, linearly independent in cohomology, satisfying $w_{i} \wedge w_{j}=0$ on the level of forms for each $i$ and $j$. Let

$$
\omega=\sum w_{j} X_{j} \in E^{1}(M) \otimes \mathbf{R}\left\langle\left\langle X_{1}, \ldots, X_{r}\right\rangle\right\rangle .
$$

The curvature vanishes so that we get a holonomy homomorphism

$$
\Theta: \pi_{1}(M, x) \rightarrow \mathbf{R}\left\langle\left\langle X_{1}, \ldots, X_{r}\right\rangle\right\rangle .
$$

Choosing, as we may, loops $\gamma_{1}, \ldots, \gamma_{r}$ at $x$ such that the matrix

$$
\left(\int_{\gamma_{j}} w_{i}\right)
$$

is non singular yields $r$ elements $1+U_{1}, \ldots, 1+U_{r}$ of $\mathbf{R}\left\langle\left\langle X_{1}, \ldots, X_{r}\right\rangle\right\rangle$, where $U_{1}, \ldots, U_{r} \in I$ are linearly independent modulo $I^{2}$. It is now easy to see that these generate a free subgroup of $\mathbf{R}\left\langle\left\langle X_{1}, \ldots, X_{r}\right\rangle\right\rangle$ of rank $r$. (This is essentially a theorem of Magnus.) It follows that $\gamma_{1}, \ldots, \gamma_{r}$ generate a free subgroup of $\pi_{1}(M, x)$ of rank $r$. Using this Chen gave the first example of how the Hodge numbers $h^{p, q}=\operatorname{dim} H^{p, q}(M)$ of a compact Kähler manifold $M$ affect its fundamental group: If

THEOREM (Chen [31], [32]). Suppose that $M$ is a compact Kähler manifold.

$$
h^{1,0}>h^{2,0}+1,
$$

then $\pi_{1}(M, x)$ contains a free subgroup of rank $\geq 2$ (and hence one of countable rank). 
In fact, Chen went a lot further and developed the theory of power series connections [41], [42], a procedure for computing, from the de Rham complex of $M$, a presentation of the $J$-adic completion of the real group ring of the fundamental group of a manifold as a quotient of the power series ring $\mathbf{R}\left\langle\left\langle H_{1}(M)\right\rangle\right\rangle$ by an ideal generated by Lie elements. To understand what this means, recall that the augmentation ideal $J$ of the real group ring of a group $\pi$ is the kernel of the augmentation $\varepsilon: \mathbf{R} \pi \rightarrow \mathbf{R}$ which takes each element of $\pi$ to 1. Its powers define a topology on $\mathbf{R} \pi$ whose completion is

$$
\mathbf{R} \pi^{\wedge}=\lim _{\leftarrow} \mathbf{R} \pi / J^{n} .
$$

The group $\&\left(\mathbf{R} \pi^{\wedge}\right)$ is a Malcev group and is called the Malcev completion of $\pi_{1}(M, x)$ and its Lie algebra $\mathrm{g}\left(\mathbf{R} \pi^{\wedge}\right)$, the Malcev Lie algebra associated to $\pi_{1}(M, x)(\mathrm{cf} .[\mathrm{Q}])$.

To see why one should be able to compute $\mathbf{R} \pi_{1}(M, x)^{\wedge}$ using formal Lie theory, consider the flat $\mathbb{S}\left(\mathbf{R} \pi_{1}(M, x)^{\wedge}\right)$-bundle whose monodromy representation

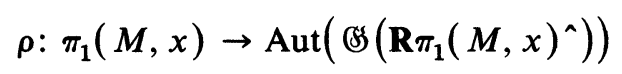

is the right regular representation $g \rightarrow\{X \rightarrow X g\}$. This bundle is trivial essentially because $\mathbb{S}\left(\mathbf{R} \pi_{1}(M, x)^{\wedge}\right)$, being the inverse limit of simply connected nilpotent Lie groups, is contractible. Choosing a trivialization then yields a $g\left(\mathbf{R} \pi_{1}(M, x)^{\hat{\Lambda}}\right)$-valued 1-form $\omega$ on $M$ whose associated transport induces the monodromy representation $\rho$ and the canonical homomorphism

$$
\pi_{1}(M, x) \rightarrow \leftrightarrow\left(\mathbf{R} \pi_{1}(M, x)^{\wedge}\right)
$$

obtained by evaluating $\rho$ at 1 .

In [41], Chen gives a direct algorithm for finding an $\mathbf{R}\left\langle\left\langle H_{1}(M)\right\rangle\right\rangle$-valued 1 -form $\omega$ and relations $R_{1}, \ldots, R_{m} \in \mathrm{g}\left(\mathbf{R}\left\langle\left\langle H_{1}(M)\right\rangle\right\rangle\right)$ such that $\omega$ is flat modulo the closed ideal generated by the $R_{j}$ 's and such that the homomorphism

$$
\Theta: \mathbf{R} \pi_{1}(M, x) \hat{\rightarrow} \mathbf{R}\left\langle\left\langle H_{1}(M)\right\rangle\right\rangle /\left(R_{1}, \ldots, R_{m}\right)
$$

induced by the transport is an isomorphism. The method is a variant of standard deformation theory. One begins with the trivial connection $(\omega=0)$ on the bundle

$$
M \times \mathbf{R}\left\langle\left\langle H_{1}(M)\right\rangle\right\rangle \rightarrow M
$$

whose monodromy representation is the augmentation

$$
\rho_{0}: \mathbf{R} \pi_{1}(M, x) \rightarrow \mathbf{R} .
$$


One proceeds to deform this connection through a sequence of connections $\left(\omega_{n}\right)_{n \geq 0}$ and simultaneously find a sequence of Lie elements $\left(R_{1}(n), \ldots, R_{1}(n)\right)$ such that the connection on (4) is flat modulo the ideal

$$
\left(R_{1}(n), \ldots, R_{1}(n)\right)+J^{n+1}
$$

and such that $T_{n}$, the transport of $\omega_{n}$, induces an isomorphism

$$
\mathbf{R} \pi_{1}(M, x) / J^{n+1} \rightarrow \mathbf{R}\left\langle\left\langle H_{1}(M)\right\rangle\right\rangle /\left(R_{1}(n), \ldots, R_{1}(n)\right)+J^{n+1} .
$$

One can interpret Chen's construction [41] of the connection form $\lim \omega_{n}$ as the construction of the dual of the versal deformation of the trivial connection on the bundle $M \times \mathbf{R} \rightarrow M$.

This method is dual to, and equivalent to, Dennis Sullivan's method of computing $\mathrm{g}\left(\mathbf{R} \pi_{1}(M, x)^{\wedge}\right)$ using the 1-minimal model of $M$ [S1], [S2]. However, Chen's method is often considerably simpler to apply, such as in the case when $M$ is a finite cell complex; the complement of a union of hyperplanes in $\mathbf{C}^{n}$ being a good example where Chen's methods work well (cf. [K]).

One of Chen's revolutionary ideas was to extend the method of power series connections to higher homotopy groups which he did in [32], [35], [38], [42]. Suppose that $M$ is a manifold which, for simplicity, we shall assume to be simply connected. Fix a point $x$ of $M$. Recall the standard isomorphism

$$
\pi_{k+1}(M, x) \cong \pi_{k}\left(\Omega_{x} M, \eta_{x}\right)
$$

where $\Omega_{x} M$ denotes the space of piecewise smooth loops in $M$ based at $x$ and $\eta_{x}$ the constant loop at $x$. By a well known theorem of Borel and Serre, the Hurewicz homomorphism induces an injection

$$
\pi_{k}\left(\Omega_{x} M, \eta_{x}\right) \otimes \mathbf{R} \rightarrow H_{k}\left(\Omega_{x} M, \mathbf{R}\right) .
$$

Multiplication of loops induces an associative product on the loop space homology. Regarding $H .\left(\Omega_{x} M, \mathbf{R}\right)$ as a graded Lie algebra with the standard bracket

$$
[U, V]=U V-(-1)^{\operatorname{deg} U \operatorname{deg} V} V U,
$$

$\pi_{k}\left(\Omega_{x} M, \eta_{x}\right) \otimes \mathbf{R}$ inherits the structure of a graded Lie algebra. From the point of view of Chen's work, the relation between $\pi \cdot\left(\Omega_{x} M, \eta_{x}\right) \otimes \mathbf{R}$ and $H .\left(\Omega_{x} M, \mathbf{R}\right)$ is analogous to the relation between $g\left(\mathbf{R} \pi_{1}(M, x)^{\hat{n}}\right)$ and $\mathbf{R} \pi_{1}(M, x)$. (The latter is naturally isomorphic to $H_{0}\left(\Omega_{x} M, \mathbf{R}\right)$.) Chen's bold step was to apply formal Lie theory to compute the Lie algebra $\pi \cdot\left(\Omega_{x} M, \eta_{x}\right)$ $\otimes \mathbf{R}$ in a way analogous to his power series method for computing the Lie algebra $\mathfrak{g}\left(\mathbf{R} \pi_{1}(M, x) \hat{)}\right)$ in the non-simply connected case. The analogue of the 
completed group ring being the completion $\mathfrak{A}$ of $H .\left(\Omega_{x} M, \mathbf{R}\right)$ with respect to its augmentation ideal:

$$
\mathfrak{U}=\prod H_{k}\left(\Omega_{x} M, \mathbf{R}\right) .
$$

The analogue of $\leftrightarrow\left(R \pi_{1}(M, x)^{\wedge}\right)$ being the generalized "group"

$$
\left\{X \in \mathfrak{A}: \log X \in \pi \cdot\left(\Omega_{x} M, \eta_{x}\right) \otimes \mathbf{R}\right\},
$$

which has a (generally non-associative) multiplication defined using the Baker-Campbell-Hausdorff formula.

Abstractly, one can consider the tensor algebra $A=\mathbf{R}\langle H\rangle$ of a graded vector space $H$. (In the sequel $H$ will be concentrated in degrees $\leq-1$.) This is a graded algebra $\sum A^{n}$, where

$$
A^{n}=\sum A^{r, n-r},
$$

and $A^{r, s}$ consists of those elements of $\otimes^{r} A$ of degree $s$. (Thus, for example, the element $a_{1} \otimes \cdots \otimes a_{s}$ has degree $s+$ the sum of the degrees of the $a_{j}$.) The elements of finite degree $A^{\hat{}}=\mathbf{R}\langle\langle H\rangle\rangle$ in the completion of $A$ with respect to the augmentation ideal $(H)$ can be viewed as a Lie algebra with bracket

$$
[U, V]=U V-(-1)^{\operatorname{deg} U \operatorname{deg} V} V U .
$$

Denote by $\mathbf{L}(H)$ the Lie sub algebra of $A^{\wedge}$ generated by $H$ and its closure in $A^{\wedge}$ by $\mathrm{g}\left(A^{\wedge}\right)$. As in the ungraded case, we can define a complete graded Lie algebra $\mathrm{g}\left(A^{\wedge} / I\right) \cong A^{\wedge} / I$ whenever $I$ is a closed ideal of $A^{\wedge}$ generated by elements of $\mathrm{g}\left(A^{\wedge}\right)$. We shall call such a Lie algebra a generalized Malcev Lie algebra.

The analogue of a connection on a trivialized bundle $M \times \mathfrak{g}\left(A^{\wedge} / I\right) \rightarrow$ $\mathfrak{g}\left(A^{\wedge} / I\right)$ is a connection form, an element $\omega$ of degree 1 in

$$
E^{*}(M) \hat{\otimes} \mathfrak{g}\left(A^{\wedge} / I\right)=\lim E^{*}(M) \otimes\left[\mathfrak{g}\left(A^{\wedge} / I\right) /\left(J^{n} \cap \mathfrak{g}\left(A^{\wedge} / I\right)\right)\right] .
$$

Such a connection form is defined to be integrable if it satisfies the usual integrability condition

$$
d \omega+1 / 2[\omega, \omega]=0
$$

Associated to each connection form is its transport map

$$
T: C .(P M) \rightarrow A^{\wedge} / I,
$$

a graded $\mathbf{R}$-linear map from the smooth chains on the path space into $A^{\wedge} / I$. It 
is defined by evaluating the $A^{\wedge} / I$-valued interated integral of degree 0

$$
1+\int \omega+\int \omega \omega+\int \omega \omega \omega+\cdots
$$

on each chain. When $\omega$ is integrable, its transport induces a chain map

$$
T: C .\left(\Omega_{x} M\right) \rightarrow A^{\wedge} / I,
$$

and holonomy maps

$$
\Theta: H .\left(\Omega_{x} M\right) \rightarrow A^{\wedge} / I, \quad \log \Theta: \pi \cdot t\left(\Omega_{x} M\right) \rightarrow \mathrm{g}\left(A^{\wedge} / I\right) .
$$

The latter map, being the composition of the logarithm of $\Theta$ with the Hurewicz homomorphism, is the analogue of the composite

$$
\pi_{1}(M, x) \rightarrow \mathscr{S}\left(\mathbf{R} \pi_{1}(M, x)^{\wedge}\right) \rightarrow \mathfrak{g}\left(\mathbf{R} \pi_{1}(M, x)^{\wedge}\right)
$$

of the natural map with the logarithm.

As in the case of the fundamental group, Chen [32], [34] used these ideas to detect large subalgebras of $\pi_{k}\left(\Omega_{x} M, \eta_{x}\right)$ : Suppose that $w_{1}, \ldots, w_{r}$ are closed differential forms on a manifold $M$, each of degree $\geq 2$, linearly independent in cohomology, satisfying $w_{i} \wedge w_{j}=0$ on the level of forms for each $i$ and $j$. Consider the graded algebra

$$
A=\mathbf{R}\left\langle X_{1}, \ldots, X_{n}\right\rangle,
$$

where $X_{j}$ has degree $1-\operatorname{deg} w_{j}$. (This is isomorphic to the tensor algebra on the graded vector space spanned by indeterminates $X_{j}$ of degree $-\operatorname{deg} w_{j}$. As each $X_{j}$ has degree $\leq-2, A$ is complete.) The form

$$
\omega=\sum w_{j} X_{j} \in E^{\cdot}(M) \otimes \mathbf{R}\left\langle X_{1}, \ldots, X_{r}\right\rangle
$$

has degree 1 and is integrable. It thus defines a holonomy homomorphism

$$
\log \Theta: \pi .\left(\Omega_{x} M\right) \rightarrow g\left(\mathbf{R}\left\langle X_{1}, \ldots, X_{r}\right\rangle\right)=\mathbf{L}\left(X_{1}, \ldots, X_{r}\right) .
$$

If there are elements $\alpha_{1}, \ldots, \alpha_{r} \in \pi_{k}\left(\Omega_{x} M, \eta_{x}\right)$ such that the matrix

$$
\left(\int_{\alpha_{j}} w_{i}\right)
$$

is non-singular, then the elements $\log \Theta\left(\alpha_{1}\right), \ldots, \log \Theta\left(\alpha_{r}\right)$ generate a free Z-Lie subalgebra of $\mathbf{L}\left(X_{1}, \ldots, X_{r}\right)$ by the graded analogue of Magnus's 
Theorem. It then follows that $\alpha_{1}, \ldots, \alpha_{r}$ generate a free Lie sub algebra of $\pi_{k}\left(\Omega_{x} M, \eta_{x}\right)$.

Chen developed this idea into the method of formal power series connections, a method for computing the Lie algebra $\pi \cdot\left(\Omega_{x} M, \eta_{x}\right) \otimes \mathbf{R}$ and the Hopf algebra $H .\left(\Omega_{x} M, \mathbf{R}\right)$ directly from the de Rham complex of $M$. As in the case of the fundamental group, this approach to de Rham homotopy theory is dual to and equivalent to Sullivan's method of minimal models.

Apart from generalizing connections by allowing the connection form to take values in a graded Lie algebra, Chen also allowed the graded Lie algebra to have a differential. A formal power series connection on $M$ is a 1-form $\omega$ on $M$ taking values in a generalized Malcev Lie algebra $g$ with a continuous differential $\delta: \mathfrak{g} \rightarrow \mathfrak{g}$ of degree -1 . The connection $(\omega, \delta)$ is integrable if

$$
D \omega+1 / 2[\omega, \omega]=0,
$$

where $D$ is the differential $d \otimes 1+1 \otimes \delta$ of the differential graded Lie algebra $E^{*}(M) \hat{\otimes}$ g. The transport

$$
T=1+\int \omega+\int \omega \omega+\int \omega \omega \omega+\cdots
$$

of a formal power series connection is then the chain map

$$
T: C .\left(\Omega_{x} M\right) \rightarrow U g
$$

obtained by integrating $T$ over chains. This induces a Hopf algebra homomorphism

$$
\Theta: H .\left(\Omega_{x} M\right) \rightarrow H .(U g) .
$$

Chen's fundamental theorem is:

THEOREM [41], [42]. For each connected manifold $M$, there exists a continuous differential $\delta$ on $\mathfrak{g}(\mathbf{R}\langle\langle H .(M)\rangle\rangle)$ of degree -1 and a $\mathfrak{g}(\mathbf{R}\langle\langle H .(M)\rangle\rangle)-$ valued 1-form $\omega$ such that $(\omega, \delta)$ is integrable. Moreover, if $M$ is simply connected, then the holonomy map

$$
\Theta: H .\left(\Omega_{x} M\right) \rightarrow H .(U \mathfrak{g}(\mathbf{R}\langle\langle H .(M)\rangle\rangle), \delta)
$$

is a graded Hopf algebra isomorphism. If $M$ is not simply connected, then the homomorphism

$$
\mathbf{R} \pi(M, x) \cong H_{0}\left(\Omega_{x} M\right) \rightarrow H_{0}(U g(\mathbf{R}\langle\langle H .(M)\rangle\rangle), \delta)
$$

is the J-adic completion of the group ring of the fundamental group as a Hopf algebra. 
Applying standard algebra and topology, one obtains the following consequence.

COROLlaRY. If $(\omega, \delta)$ is an integrable connection as in the theorem, then, when $M$ is simply connected, the logarithm of the transport of $\omega$ induces a Lie algebra isomorphism

$$
\log \Theta: \pi \cdot\left(\Omega_{x} M\right) \rightarrow H \cdot(g(\mathbf{R}\langle\langle H .(M)\rangle\rangle), \delta) .
$$

When $M$ is not simply connected, the logarithm of $T$ induces an isomorphism of Malcev Lie algebras

$$
\log \Theta: \mathfrak{g}(\mathbf{R} \pi(M, x)) \rightarrow H_{0}(\mathfrak{g}(\mathbf{R}\langle\langle H .(M)\rangle\rangle), \delta) .
$$

One could not develop such ideas without pondering the relationship between iterated integrals and the cohomology of the loop space of $M$. This Chen did in a series of papers [35], [39], [42] interwoven with those in which he developed formal power series connections. Regarding iterated integrals as a subspace $\mathscr{J} \cdot(P M)$ of the de Rham complex $E^{\circ}(P M)$ of the path space of $M$, Chen showed that $\mathscr{J} \cdot(P M)$ is a differential graded algebra with product and differential induced from those of $E^{*}(P M)$, and gave a completely algebraic description of $\mathscr{J} \cdot(P M)$ as the reduced bar construction on the de Rham complex of $M$, a useful variant of the classical bar construction [40]. Denote the complex of iterated integrals restricted to the loop space $\Omega_{x} M$ by $\mathscr{J} \cdot\left(\Omega_{x} M\right)$. He also showed that the homomorphism

$$
\mu: E^{\cdot}\left(\Omega_{x} M\right) \rightarrow E^{\cdot}\left(\Omega_{x} M \times \Omega_{x} M\right)
$$

induced by multiplication of paths induces a coproduct

$$
\begin{aligned}
\Delta: \mathscr{J} \cdot\left(\Omega_{x} M\right) & \rightarrow \mathscr{J} \cdot\left(\Omega_{x} M\right) \otimes \mathscr{J} \cdot\left(\Omega_{x} M\right) \\
\int w_{1} w_{2} \ldots w_{r} & \rightarrow \sum \int w_{1} \ldots w_{i} \otimes \int w_{i+1} \ldots w_{r} .
\end{aligned}
$$

With this coproduct, $\mathscr{J} \cdot\left(\Omega_{x} M\right)$ becomes a $d . g$. Hopf algebra. Integration induces a graded Hopf algebra homomorphism

$$
\mathbf{I}: H^{\bullet}\left(\mathscr{J} \cdot\left(\Omega_{x} M\right)\right) \rightarrow H^{\bullet}\left(\Omega_{x} M, \mathbf{R}\right)
$$

Naively one may expect that $\mathbf{I}$ is always an isomorphism. However, if we filter iterated integrals by length:

$$
\mathbf{R} \subseteq \mathscr{J}_{1} \subseteq \mathscr{J}_{2} \subseteq \cdots \subseteq \mathscr{J} \cdot\left(\Omega_{x} M\right)
$$


then standard properties of iterated integrals imply that, as a $\pi_{1}(M, x)$ module, $H^{*}\left(\mathscr{J} \cdot\left(\Omega_{x} M\right)\right)$ is a union of the unipotent submodules

$$
\operatorname{im}\left\{H^{\bullet}\left(\mathscr{J}_{s}\right) \rightarrow H^{\bullet}\left(\mathscr{J} \cdot\left(\Omega_{x} M\right)\right)\right\} .
$$

So I cannot be an isomorphism in general. However, in the absence of the fundamental group, Chen proved the following result which is a de Rham analogue of Adams fundamental theorem $[A]$.

THEOREM [35], [38], [42]. If $M$ is simply connected, then the integration map I is a graded Hopf algebra isomorphism.

In the non-simply connected case, he proved the following result which is related to work of Stallings [S]. An elementary proof of it may be found in $[\mathrm{H}]$.

THEOREM [39]. For all $s \geq 0$, the homomorphism

$$
H^{0}\left(\mathscr{J}_{s}\right) \rightarrow \operatorname{Hom}\left(\mathbf{Z} \pi_{1}(M, x) / J^{s+1}, \mathbf{R}\right)
$$

induced by integration, is an isomorphism. Consequently, if $\pi_{1}(M, x)$ is finitely generated, then the adjoint of the integration map

$$
\mathbf{R} \pi(M, x)^{\wedge} \rightarrow \operatorname{Hom}\left(H^{0}\left(\mathscr{J} \cdot\left(\Omega_{x} M\right)\right), \mathbf{R}\right)
$$

is an isomorphism of complete Hopf algebras.

In his later papers [42], [43], [47], [54], Chen studied the de Rham theory of spaces $P_{f}$ obtained by pulling back the fibration $P M \rightarrow M \times M$ along a function $f: N \rightarrow M \times M$ :

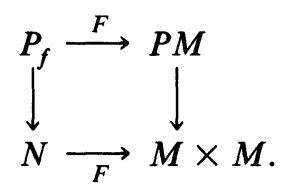

Chen considered the subcomplex $\mathscr{J} \cdot\left(P_{f}\right)$ of $E^{*}\left(P_{f}\right)$ generated by pullbacks of iterated integrals along $F$ and pullbacks of forms on $N$ along the projection. This complex can be described as the circular bar construction [45] on the de Rham complexes of $N$ and $M \times M$. Chen proved that the cohomology of $\mathscr{J} \cdot\left(P_{f}\right)$ is isomorphic to $H^{*}\left(P_{f}\right)$ when, for example, $M$ is simply connected. Taking $N=M$ and $f$ to be the diagonal, Chen obtained a complex for computing the cohomology of the free loop space of $M$, anticipating the recent work on cyclic homology and the cohomology of the free loop space. 


\section{REFERENCES}

[A] J.F. ADAMS, On the cobar construction, Colloque de topologie algébrique (Louvain, 1956), George Thone, Liège, Masson, Paris, 1957, pp. 81-87.

[H] R. HAIN, The geometry of the mixed Hodge structure on the fundamental group, Algebraic Geometry, 1985, Proc. Symp. Pure Math., vol. 46 (1987), pp. 247-282.

[K] T. KoHNo, On the holonomy Lie algebra and the nilpotent completion of the fundamental group of the complement of hypersurfaces, Nagoya J. Math., vol. 92 (1983), pp. 21-37.

[M] J. Milnor, Isotopy of links, A Symposium in Honor of S. Lefschetz 1954, Princeton University Press, 1957, pp. 280-305.

[Q] D. Quillen, Rational homotopy theory, Ann. of Math., vol. 90 (1969), pp. 205-295.

[S] J. Stallings, Quotients of the powers of the augmentation ideal in a group ring. In: L. Neuwirth (ed.), Knots, Groups and 3-manifolds, Papers dedicated to the memory of R.H. Fox, Annals of Math. Studies 84, Princeton, 1975.

[S1] D. Sullivan, Topology of manifolds and differential forms, Proceedings of a conference on manifolds, Tokyo, 1973.

[S2] _. Infinitesimal computations in topology, Publ. Math. IHES., vol. 47 (1977), pp. 269-331.

Richard Hain

Philippe Tondeur

April 1989 


\title{
Students of Kuo-Tsai Chen
}

\author{
Gerald John Ciaccai, 1970 \\ Tryggve Fossum, 1972 \\ Salma Shukrallah Wanna, 1976 \\ Larry James Williams, 1976 \\ John Lawrence Cuadrado, 1977 \\ Richard Hain, 1980
}

\section{Publications of Kuo-Tsai Chen}

1. Integration in free groups, Ann. of Math., vol. 54 (1951), pp. 147-162.

2. Commutator calculus and link invariants, Proc. Amer. Math. Soc., vol. 3 (1952), pp. 44-55.

3. Isotopy invariants of links, Ann. of Math., vol. 56 (1952), pp. 343-353.

4. A group ring method for infinitely generated groups, Trans. Amer. Math. Soc., vol. 76 (1954), pp. 275-287.

5. Interated integrals and exponential homomorphisms, Proc. London Math. Soc., vol. 4 (1954), pp. 502-512.

6 On the composition functions of nilpotent Lie groups, Proc. Amer. Math. Soc., vol. 8 (1957), pp. 1158-1159.

7. Integration of paths, geometric invariants and generalized Baker-Hausdorff formula, Ann. of Math., vol. 65 (1957), pp. 163-178.

8. Integration of paths, a faithful representation of paths by noncommutative formal power series, Trans, Amer. Math. Soc., vol. 89 (1958), pp. 395-407.

9. Exponential isomorphism for vector spaces and its connection with Lie groups, J. London Math. Soc., vol. 33 (1958), pp. 170-177.

10. Free differential calculus IV (With R.H. Fox and R.C. Lyndon), Ann. of Math., vol. 68 (1958), pp. 81-97.

11. Linear independence of exponentials of Lie elements, An. Acad. Brasil. Cienc., vol. 31 (1959), pp. 507-509.

12. Formal differential equations, Ann. of Math., vol. 73 (1961), pp. 110-133.

13. Decomposition of differential equations, Math. Ann., vol. 146 (1962), pp. 263-278.

14. An expansion formula for differential equations, Bull. Amer. Math. Soc., vol. 68 (1962), pp. 341-344.

15. Decomposition and equivalence of local vector fields, Proc. Nat. Acad. Sci., vol. 49 (1963), pp. 740-741.

16. Expansion of solutions of differential systems, Arch. Rational Mech. Anal., vol. 13 (1963), pp. 348-363.

17. On local diffeomorphisms about an elementary fixed point, Bull. Amer. Math. Soc., vol. 69 (1963), pp. 838-840.

18. Equivalence and decomposition of vector fields about an elementary critical point, Amer. J. Math., vol. 85 (1963), pp. 693-722.

19. Local diffeomorphisms $-C^{\infty}$ realization of formal properties, Amer. J. Math., vol. 87 (1965), pp. 140-157.

20. On a generalization of Picard's approximation, J. Differential Equations, vol. 2 (1966), pp. 438-448.

21. On nonelementary hyperbolic fixed points of diffeomorphisms, Proceedings of International Symposium on Differential Equations and Dynamic Systems, Academic Press, San Diego, 1967 , pp. 525-530.

22. Iterated path integrals and generalized paths, Bull. Amer. Math. Soc., vol. 73 (1967), pp. 935-938. 
23. Algebraization of iterated integration along paths, Bull. Amer. Math. Soc., vol. 73 (1967), pp. 975-978.

24. Normal forms of local diffeomorphism on the real line, Duke. Math. J., vol. 35 (1968), pp. 549-556.

25. Algebraic paths, J. Algebra, vol. 10 (1968), pp. 8-36.

26. Homotopy of algebras, J. Algebra, vol. 10 (1968), pp. 183-193.

27. Convering-space-like algebras, J. Algebra, vol. 13 (1969), pp. 308-326.

28. An algebraic dualization of fundamental groups, Bull. Amer. Math. Soc., vol. 75 (1969), pp. 1020-1024.

29. An exact dynamical system is tree-like and vice versa, Trans. Amer. Math. Soc., vol. 149 (1970), pp. 561-567.

30. A sufficient condition for nonabelianness of fundamental groups of differential manifolds, Proc. Amer. Math. Soc., vol. 26 (1970), pp. 196-198.

31. Algebras of iterated path integrals and fundamental groups, Trans. Amer. Math. Soc., vol. 156 (1971), pp. 359-379.

32. Differential forms and homotopy groups, J. Differential Geom., vol. 6 (1971), pp. 231-246.

33. On Whitehead products, Proc. Amer. Math. Soc., vol. 34 (1972), pp. 257-259.

34. Free subalgebras of loop space homology and Massey products, Topology, vol. 11 (1972), pp. 237-243.

35. Iterated integrals of differential forms and loop space homology, Ann. of Math., vol. 97 (1973), pp. 217-246.

36. Fundamental groups, nilmanifolds and iterated integrals, Bull. Amer. Math. Soc., vol. 79 (1973), pp. 1033-1035.

37. Solvability on manifolds by quadratures, Bull. Amer. Math. Soc., vol. 80 (1974), pp. 1210-1212.

38. Connection, holonomy and path space homology, Proceedings of Symposia in Pure Mathematics, vol. 27, pp. 39-52, American Mathematical Society, Providence, R.I., 1975.

39. Iterated integrals, fundamental groups and covering spaces, Trans. Amer. Math. Soc., vol. 206 (1975), pp. 83-98.

40. "Reduced bar construction on de Rham complex" in A Collection of papers in honor of Samuel Eilenberg, Academic Press, San Diego, 1976, pp. 19-32.

41. Extension of $C^{\infty}$ function algebra by integrals and Malcev completion of $\pi_{1}$, Adv. in Math., vol. 23 (1977), pp. 181-210.

42. Iterated path integrals, Bull. Amer. Math. Soc., vol. 83 (1977), pp. 831-879.

43. Pullback de Rham cohomology of the free path fibration, Trans. Amer. Math. Soc., vol. 242 (1978), pp. 307-318.

44. Path space differential forms and transports of connections, Bull. Inst. Math. Acad. Sinica, vol. 6 (1978), pp. 457-477.

45. Circular bar constructions, J. Algebra, vol. 57 (1979), pp. 466-483.

46. Poles of maps into $P_{n}(C)$ and Whitehead integrals, South Asian Bull. Math. vol. 3 (1979), pp. 116-124.

47. Pullback path fibration, homotopies and iterated integrals, Bull. Inst. Math. Acad. Sinica, vol. 8 (1980), pp. 263-275.

48. The Euler operator, Arch. Rational mech. Anal., vol. 75 (1981), pp. 175-191.

49. On the Hopf Index theorem and the Hopf invariant, Bull. Amer. Math. Soc., vol. 5 (19,81), pp. 57-69.

50. "Pairs of maps into complex projective space" in Contribution to analysis and geometry, Johns Hopkins Press, Baltimore, Maryland, 1980, pp. 51-62.

51. Degeneracy indices and Chern Classes, Adv. in Math., vol. 45 (1982), pp. 73-91.

52. On the Bezout theorem, Amer. J. Math., vol. 106 (1984), pp. 725-744.

53. "Loop spaces and differential forms" in Homotopie Algébrique et Algèbre Locale, Astérisque, vols. 113-114, 1984, pp. 725-744.

54. Smooth maps, pullback path spaces, connections and torsions, Trans. Amer. Math. Soc., vol. 297 (1986), pp. 617-627. 